\title{
(R)- $\alpha$-Aminoadipic Acid: A Versatile Precursor for the Synthesis of D-Amino Acids
}

\author{
Amina Sadiq and Norbert Sewald \\ Department of Chemistry, Bielefeld University, Universitätsstraße 25, 33615 Bielefeld, Germany \\ Correspondence should be addressed to Norbert Sewald; norbert.sewald@uni-bielefeld.de
}

Received 26 February 2013; Accepted 27 August 2013

Academic Editor: Sambasivarao Kotha

Copyright ( 2013 A. Sadiq and N. Sewald. This is an open access article distributed under the Creative Commons Attribution License, which permits unrestricted use, distribution, and reproduction in any medium, provided the original work is properly cited.

The ready accessibility of $(R)$ - $\alpha$-aminoadipic acid by enzymatic cleavage of cephalosporin $C(C e p h C)$ in the production of 7 aminocephalosporanic acid (7-ACA) on a large scale makes it a favorable chiral pool building block for the synthesis of unusual amino acids. A route for the synthesis of C-5-alkenyl and C-6-alkylidene derivatives of $(R)$-pipecolic acid is described which utilizes $(R)-\alpha$-aminoadipic acid as the enantiomerically pure starting material. Moreover, the synthesis of azido and triazolyl derivatives of $(R)$ - $\alpha$-aminoadipic acid is reported.

\section{Introduction}

$\alpha$-Amino acids, one of the important classes of natural products, play fundamental roles in chemistry and biology. In addition to their vital roles as building block of proteins and as intermediates in metabolism, they also constitute a broad array of chiral pool building blocks and organocatalysts [13]. Their ready availability, low cost, and high enantiomeric purity make them valuable starting materials for the synthesis of unusual amino acids. D-Amino acids are far less abundant in nature in contrast to L-configured counterparts. In addition, D-amino acids display interesting conformational features; for example, they stabilize turn conformations in peptides [4].

$\alpha$-Aminoadipic acid is a six-carbon analog of aspartic and glutamic acid which is found in plants and microorganisms. It is a metabolite in the principal biochemical pathway of lysine [5]. During the past decades, $\alpha$-aminoadipic acid has received attention from chemists active in the areas of peptide chemistry, organic synthesis, biosynthesis, and neuroscience. $(R)$ - $\alpha$-Aminoadipic acid exhibits a selective antagonistic activity at the $N$-methyl- $D$-aspartate subtype of glutamate receptors $[6,7]$. It has also been used in the synthesis of carbocyclic nucleoside precursors [8]. (R)- $\alpha$-Aminoadipic acid $\mathbf{1}$ is a constituent of cephalosporin $\mathrm{C}$ and penicillin
$N$. In the pharmaceutical semisynthesis of cephalosporin derivatives, the central intermediate 7-aminocephalosporanic acid (7-ACA) is obtained by enzymatic cleavage of the fermentation product cephalosporin $\mathrm{C}$ (CephC) [9]. As the enantiomerically pure $(R)$ - $\alpha$-aminoadipic acid is available from this process on a large scale, we embarked on a project to explore the application of this chiral pool building block for the synthesis of $(R)$-pipecolic acid and its derivatives [10,11].

Pipecolic acid, also known as homoproline or piperidine2-carboxylic acid, a six-carbon natural nonproteinogenic $\alpha$-amino acid, is an intermediate of lysine metabolism in various organisms including bacteria, yeast, fungi, and mammals. (S)-Pipecolic acid is an important precursor of several bioactive compounds such as synthetic peptides [12], local anesthetics [13], and potential enzyme inhibitors [14, 15] and is a component of biologically important natural products such as the immunomodulators rapamycin [16], the immunosuppressant FK506 [17], the antitumor antibiotic sandramycin [18], and the anticancer agent VX710 [19]. It also occurs in the efrapeptins and neoefrapeptins, ATPase inhibiting peptide families [20]. (R)-Pipecolic acid is present in the histone deacetylase inhibitors trapoxin $\mathrm{A}$ and apicidin [21]. These applications of pipecolic acid stimulated us to synthesize further derivatives of pipecolic acid which could be further employed in peptide modifications. 


\section{Material and Methods}

All reactions were carried out in oven-dried glassware with magnetic stirrers under an argon atmosphere. THF was dried over $\mathrm{Na}$ /benzophenone and DCM was dried over $\mathrm{CaH}_{2}$. Commercially available chemicals were purchased from Sigma-Aldrich and Alfa Aesar. EtOAc and n-pentane were distilled before use. Flash chromatography was carried out using silica gel, particle size $0.035-0.070 \mathrm{~mm}$. Specific rotation of synthesized compounds was recorded on a Jasco DIP-366 digital polarimeter. ${ }^{1} \mathrm{H}$ NMR spectra were recorded on a $500 \mathrm{MHz}$ Bruker DRX spectrometer in $\mathrm{CDCl}_{3}$ (unless otherwise stated) referenced relative to residual $\mathrm{CHCl}_{3}(\delta=$ $7.26 \mathrm{ppm}$ ). Chemical shifts are reported in ppm and coupling constants in Hertz. ${ }^{13} \mathrm{C}$ NMR spectra were recorded on a $500 \mathrm{MHz}$ Bruker DRX (125.7 MHz) with proton decoupling. EI and CI mass spectra (including EI accurate mass measurements) were recorded using an Autospec X magnetic sector mass spectrometer with EBE geometry (Vacuum Generators, Manchester, UK) equipped with a standard EI or CI source. Samples were introduced by push rod in aluminium crucibles if not otherwise noted. Ions were accelerated by $8 \mathrm{kV}$ in EI mode and $6 \mathrm{kV}$ in CI mode. Accurate mass measurement experiments with ESI or MALDI were performed using a fourier transform ion cyclotron resonance mass spectrometer APEX III (Bruker Daltonik GmbH, Bremen, Germany) equipped with a $7.0 \mathrm{~T}, 160 \mathrm{~mm}$ bore superconducting magnet (Bruker Analytik GmbH-Magnetics, Karlsruhe, Germany), infinity cell, and interfaced with an external (nano)ESI or MALDI ion source. Nitrogen served both as the nebulizer gas and the dry gas for ESI. Nitrogen was generated by a Bruker nitrogen generator NGM 11. Argon served as a cooling gas in the infinity cell and a collision gas for $\mathrm{MS}^{n}$ experiments.

Benzyl (R)-2-(Benzyloxycarbonylamino)-6-(methylsulfonyloxy)hexanoate (7). $\mathrm{Et}_{3} \mathrm{~N}$ (1.68 mmol, $\left.2.5 \mathrm{eq} ., 0.23 \mathrm{~mL}\right)$ and methanesulfonyl chloride $(0.81 \mathrm{mmol}, 1.2 \mathrm{eq}$., $0.06 \mathrm{~mL})$ were added to a solution of $4(0.673 \mathrm{mmol}, 1$ eq., $250 \mathrm{mg})$ in dry $\mathrm{CH}_{2} \mathrm{Cl}_{2}(8 \mathrm{~mL})$ at $0^{\circ} \mathrm{C}$, and the mixture was stirred at the same temperature. The progress of the reaction was monitored by TLC. After the reaction was complete ( $\sim 5 \mathrm{~min})$, it was quenched by addition of water $(10 \mathrm{~mL})$. The mixture was then extracted with $\mathrm{CH}_{2} \mathrm{Cl}_{2}(3 \times 15 \mathrm{~mL})$, and the combined organic phase was washed with brine $(15 \mathrm{~mL})$ and dried over anhydrous $\mathrm{Na}_{2} \mathrm{SO}_{4}$. Evaporation of solvent and silica gel column chromatography of the resulting residue (n-pentane/EtOAc 6:4) gave the corresponding mesylate 7 (270 $\mathrm{mg}, 92 \%)$ as a colorless oil.

$[\alpha]_{\mathrm{D}}{ }^{23}=+23.0\left(c=1.5, \mathrm{CHCl}_{3}\right) .{ }^{1} \mathrm{H} \mathrm{NMR}(500 \mathrm{MHz}$, $\left.\mathrm{CDCl}_{3}\right): \delta=7.41-7.33(\mathrm{~m}, 10 \mathrm{H}, \mathrm{Ar}-\mathrm{H}), 5.52$ (br d, $1 \mathrm{H}, J=$ $8.3 \mathrm{~Hz}, \mathrm{NH}), 5.24-5.15\left(\mathrm{~m}, 2 \mathrm{H}, \mathrm{Bn}-\mathrm{CH}_{2}\right), 5.12(\mathrm{~s}, 2 \mathrm{H}, \mathrm{Bn}-$ $\left.\mathrm{CH}_{2}\right), 4.39(\mathrm{~m}, 1 \mathrm{H}, \mathrm{CH}), 4.17-4.13\left(\mathrm{~m}, 2 \mathrm{H}, \mathrm{CH}_{2}\right), 2.95(\mathrm{~s}, 3 \mathrm{H}$, $\left.\mathrm{CH}_{3}\right), 1.87\left(\mathrm{~m}, 1 \mathrm{H}, \mathrm{CH}_{2}\right), 1.83-1.66\left(\mathrm{~m}, 3 \mathrm{H}, \mathrm{CH}_{2}\right), 1.51-1.35(\mathrm{~m}$, $\left.2 \mathrm{H}, \mathrm{CH}_{2}\right) .{ }^{13} \mathrm{C} \mathrm{NMR}\left(125.7 \mathrm{MHz}, \mathrm{CDCl}_{3}\right): \delta=172.0$ (ㄷO), 155.9 (NCOO), 136.6 (Ar- $\underline{\mathrm{C}}), 135.2$ (Ar- $\underline{\mathrm{C}}$ ), 128.6 (10 A $\overline{\mathrm{r}}-\underline{\mathrm{CH}}$ ), $69.4\left(\mathrm{OC}_{2}\right), 67.2\left(\mathrm{Bn}-\underline{\mathrm{CH}}_{2}\right), 67.0\left(\mathrm{Bn}-\overline{\mathrm{CH}}_{2}\right), 53.6(\underline{\mathrm{CH}}), 37.2$ $\left(\underline{\mathrm{CH}}_{3}\right), 31.9\left(\underline{\mathrm{CH}}_{2}\right), 28.5\left(\underline{\mathrm{CH}}_{2}\right), 21.2\left(\underline{\mathrm{CH}}_{2}\right) \cdot \mathrm{C}_{22} \overline{\mathrm{H}}_{27} \mathrm{NO}_{7} \mathrm{~S}$
(449.16). HRMS (ESI-FT-ICR): $m / z=472.14057$; calcd. for $\left[\mathrm{C}_{22} \mathrm{H}_{27} \mathrm{NO}_{7} \mathrm{SNa}\right]^{+}: m / z=472.14004$.

Benzyl (R)-6-Azido-2-(benzyloxycarbonylamino)hexanoate (8). $\mathrm{NaN}_{3}(1.00 \mathrm{mmol}, 1.5 \mathrm{eq}, 70 \mathrm{mg})$ was added to a solution of mesylate 7 ( $0.68 \mathrm{mmol}, 1$ eq., $300 \mathrm{mg})$ in dry DMF $(8 \mathrm{~mL})$. The reaction mixture was stirred at room temperature for $18 \mathrm{~h}$. It was then poured into water $(15 \mathrm{~mL})$ and extracted with EtOAc $(3 \times 15 \mathrm{~mL})$. The combined organic phase was washed with brine $(20 \mathrm{~mL})$ and dried over $\mathrm{Na}_{2} \mathrm{SO}_{4}$. The solvent was removed under reduced pressure and the residue thus obtained was purified by silica gel column chromatography (ether/EtOAc $7: 3)$ to give $\mathbf{8}(202 \mathrm{mg}, 76 \%)$ as a colorless oil. $[\alpha]_{\mathrm{D}}{ }^{23}=+30.1\left(c=1.5, \mathrm{CHCl}_{3}\right) \cdot{ }^{1} \mathrm{H} \mathrm{NMR}(500 \mathrm{MHz}$, $\left.\mathrm{Me}_{3} \mathrm{OD}\right): \delta=7.39-7.28$ (m, 10H, Ar-H), 5.22-5.13 (m, 2H, $\left.\mathrm{Bn}-\mathrm{CH}_{2}\right), 5.10\left(\mathrm{~s}, 2 \mathrm{H}, \mathrm{Bn}-\mathrm{CH}_{2}\right), 4.15(\mathrm{~m}, 1 \mathrm{H}, \mathrm{CH}), 3.24(\mathrm{t}$, $\left.J=6.7 \mathrm{~Hz}, 2 \mathrm{H}, \mathrm{CH}_{2}\right), 1.82\left(\mathrm{~m}, 1 \mathrm{H}, \mathrm{CH}_{2}\right) 1.67\left(\mathrm{~m}, 1 \mathrm{H}, \mathrm{CH}_{2}\right)$, 1.60-1.50 (m, 2H, CH $), 1.49-1.40\left(\mathrm{~m}, 2 \mathrm{H}, \mathrm{CH}_{2}\right) .{ }^{13} \mathrm{C} \mathrm{NMR}$ $\left(125.7 \mathrm{MHz}, \mathrm{CDCl}_{3}\right): \delta=174.9$ (COO), 159.7 (NCOO), 139.3 (Ar-ㅡ), 138.3 (Ar- $\underline{\mathrm{C}}), 128.6$ (10 Ar- $\underline{\mathrm{CH}}), 68.9\left(\underline{\mathrm{CH}}_{2}\right), 68.7$ $\left(\underline{\mathrm{CH}}_{2}\right), 56.5\left(\underline{\mathrm{CH}}_{2}\right), 53.2(\underline{\mathrm{CH}}), 33.2\left(\underline{\mathrm{CH}}_{2}\right), 30.4\left(\underline{\mathrm{CH}}_{2}\right), 25.1$ $\left(\overline{\mathrm{CH}}_{2}\right) \cdot \mathrm{C}_{21} \mathrm{H}_{24} \mathrm{~N}_{4} \mathrm{O}_{4}$ (396.18). HRMS (ESI-FT-ICR): $\mathrm{m} / z=$ 397.18724; calcd. for $\left[\mathrm{C}_{21} \mathrm{H}_{24} \mathrm{~N}_{4} \mathrm{O}_{4} \mathrm{H}\right]^{+}: m / z=397.18703$.

2.1. General Procedure for Copper Catalyzed Azide-Alkyne Cycloaddition (CuAAC). Substituted alkyne (0.352 mmol, 1 eq.) was added to a solution of azide $8(0.352 \mathrm{mmol}$, 1eq.) in a $1: 9$ mixture of DMSO $(0.5 \mathrm{~mL})$ and $\mathrm{H}_{2} \mathrm{O}$ (4.5 mL). $\mathrm{CuSO}_{4} \cdot 5 \mathrm{H}_{2} \mathrm{O}$ (0.088 mmol, 0.25 eq.) was added to the mixture, followed by the addition of sodium ascorbate $(0.160 \mathrm{mmol}, 0.5$ eq. $)$. The reaction mixture was vigorously stirred overnight at room temperature. After completion (monitored by TLC) the reaction was quenched by addition of water and extracted with $\mathrm{CH}_{2} \mathrm{Cl}_{2}(3 \times 20 \mathrm{~mL})$. The combined organic phase was washed with water and brine and concentrated in vacuo. The residue was purified by flash column chromatography (n-pentane/EtOAc $1: 1$ ) to give the product as colorless oil.

2.1.1. Benzyl (R)-2-(Benzyloxycarbonylamino)-6-(4-phenyl1H-1,2,3-triazol-1-yl)hexanoate (11). Colorless oil, yield $100 \mathrm{mg}(57 \%) \cdot[\alpha]_{\mathrm{D}}{ }^{23}=+27.3\left(c=1.5, \mathrm{CHCl}_{3}\right) .{ }^{1} \mathrm{H} \mathrm{NMR}$ $\left(500 \mathrm{MHz} \mathrm{CDCl}_{3}\right): \delta=7.82-7.76(\mathrm{~m}, 2 \mathrm{H}, \mathrm{Ar}-\mathrm{H}), 7.69$ (s, 1H, Ar-H), 7.42-7.40 (m, 2H, Ar-H), 7.35-7.30 (m, 11H, Ar-H), $5.35(\mathrm{~d}, J=8.2 \mathrm{~Hz}, 1 \mathrm{H}, \mathrm{NH}), 5.19-5.04(\mathrm{~m}, 4 \mathrm{H}$, $\left.\mathrm{Bn}-\mathrm{CH}_{2}\right), 4.43(\mathrm{~m}, 1 \mathrm{H}, \mathrm{CH}), 4.31\left(\mathrm{t}, J=7.1 \mathrm{~Hz}, 2 \mathrm{H}, \mathrm{CH}_{2}\right)$, 1.96-1.87 (m, 2H, $\left.\mathrm{CH}_{2}\right)$ 1.75-1.68 (m, 2H, $\left.\mathrm{CH}_{2}\right), 1.35(\mathrm{~m}, 1 \mathrm{H}$, $\left.\mathrm{CH}_{2}\right), 1.28\left(\mathrm{~m}, 1 \mathrm{H}, \mathrm{CH}_{2}\right) .{ }^{13} \mathrm{C} \mathrm{NMR}\left(125.7 \mathrm{MHz}, \mathrm{CDCl}_{3}\right)$ : $\delta=171.9$ (COO), 155.8 (NCOO), 147.7 (C), 136.1 (Ar-C), 135.1 (Ar- $\underline{\mathrm{C}}$ ), 130.6 (3 Ar- $\underline{\mathrm{CH}}$ ), 128.8 (12 Ar- $\underline{\mathrm{CH}}$ ), 125.7 (Ar-C), $119.4(\mathrm{CH}), 67.3\left(\mathrm{Bn}-\mathrm{CH}_{2}\right), 67.0\left(\mathrm{Bn}-\mathrm{CH}_{2}\right), 53.4(\underline{\mathrm{CH}}), 49.9$ $\left(\underline{\mathrm{CH}}_{2}\right), 32.0\left(\underline{\mathrm{CH}}_{2}\right), 29.6\left(\underline{\mathrm{CH}}_{2}\right), 22.0\left(\underline{\mathrm{CH}}_{2}\right) . \mathrm{C}_{29} \overline{\mathrm{H}}_{30} \mathrm{~N}_{4} \mathrm{O}_{4}$ (498.18). HRMS (ESI-FT-ICR): $m / z=521.21663$; calcd. for $\left[\mathrm{C}_{29} \mathrm{H}_{30} \mathrm{NO}_{4} \mathrm{Na}\right]^{+}: m / z=521.21593$.

2.1.2. Benzyl (R)-2-(Benzyloxycarbonylamino)-6-(4-ethoxycarbonyl-1H-1,2,3-triazol-1-yl)hexanoate (12). Colorless oil, yield $75 \mathrm{mg}(60 \%) \cdot[\alpha]_{\mathrm{D}}^{23}=+21.4\left(c=1.5, \mathrm{CHCl}_{3}\right)$. 
${ }^{1} \mathrm{H}$ NMR $\left(500 \mathrm{MHz}, \mathrm{CDCl}_{3}\right): \delta=8.04(\mathrm{~s}, 1 \mathrm{H}, \mathrm{CH}), 7.39-$ $7.31(\mathrm{~m}, 10 \mathrm{H}, \mathrm{Ar}-\mathrm{H}), 5.38$ (br d, $1 \mathrm{H}, J=8.2 \mathrm{~Hz}, \mathrm{NH}), 5.18-$ $5.11\left(\mathrm{~m}, 4 \mathrm{H}, \mathrm{Bn}-\mathrm{CH}_{2}\right), 4.45-4.41\left(\mathrm{~m}, 3 \mathrm{H}, \mathrm{CH}_{2}, \mathrm{CH}\right), 4.32(\mathrm{t}$, $\left.J=7.3 \mathrm{~Hz}, 2 \mathrm{H}, \mathrm{CH}_{2}\right), 1.97-1.86\left(\mathrm{~m}, 2 \mathrm{H}, \mathrm{CH}_{2}\right), 1.76-1.70(\mathrm{~m}$, $\left.2 \mathrm{H}, \mathrm{CH}_{2}\right) 1.42\left(\mathrm{t}, J=7.1 \mathrm{~Hz}, 3 \mathrm{H}, \mathrm{CH}_{3}\right), 1.37-1.26(\mathrm{~m}, 2 \mathrm{H}$, $\left.\mathrm{CH}_{2}\right) .{ }^{13} \mathrm{C}$ NMR $\left(125.7 \mathrm{MHz}, \mathrm{CDCl}_{3}\right): \delta=171.8$ ( $\left.\mathrm{COO}\right)$, 160.7 (COO), 155.8 (NCOO), 140.3 (ㅁ) , 136.1 (Ar- $\underline{\mathrm{C}}$ ), 135.1 $(\mathrm{Ar}-\mathrm{C}), 128.6(10 \mathrm{Ar}-\mathrm{CH}), 127.2(\mathrm{CH}), 67.3\left(\mathrm{Bn}-\mathrm{CH}_{2}\right), 67.1(\mathrm{Bn}-$ $\left.\mathrm{CH}_{2}\right), 61.3\left(\mathrm{COOCH}_{2}\right), 53.4(\mathrm{CH}), 50.4\left(\mathrm{CH}_{2}\right), 32.0\left(\mathrm{CH}_{2}\right)$, $29.4\left(\mathrm{CH}_{2}\right), 21.9\left(\underline{\mathrm{CH}}_{2}\right), 14.3\left(\underline{\mathrm{CH}}_{3}\right) . \mathrm{C}_{26} \mathrm{H}_{30} \mathrm{~N}_{4} \mathrm{O}_{6}(494.22)$. HRMS (EI): $m / z=494.21457$; calcd. for $\left[\mathrm{C}_{26} \mathrm{H}_{30} \mathrm{~N}_{4} \mathrm{O}_{6}\right]^{+}$: $m / z=494.21599$.

2.2. General Procedure for Ruthenium Catalyzed AzideAlkyne Cycloaddition (RuAAC). A mixture of azide $\mathbf{8}$ (0.252 mmol, 1 eq., $100 \mathrm{mg})$, alkyne $(0.504 \mathrm{mmol}, 2$ eq. $)$, and $\mathrm{Cp}^{*} \mathrm{RuCl}\left(\mathrm{PPh}_{3}\right)_{2}$ (0.005 mmol, 0.02 eq. $)$ in benzene $(7 \mathrm{~mL})$ was stirred under reflux for $24 \mathrm{~h}$. The progress of the reaction was monitored by TLC. After completion of reaction, the solvent was evaporated under vacuum, and the product was purified by silica gel chromatography (n-pentane/EtOAc $1: 1$ ) to give the product as colorless oil.

2.2.1. (R)-Benzyl 2-(Benzyloxycarbonylamino)-6-(5-phenyl1H-1,2,3-triazol-1-yl)hexanoate (9). Colorless oil, yield $80 \mathrm{mg}$ $(64 \%) .[\alpha]_{\mathrm{D}}{ }^{23}=+27.8\left(c=1.5, \mathrm{CHCl}_{3}\right) .{ }^{1} \mathrm{H} \mathrm{NMR}(500 \mathrm{MHz}$, $\left.\mathrm{CDCl}_{3}\right): \delta=7.69(\mathrm{~s}, 1 \mathrm{H}, \mathrm{Ar}-\mathrm{H}), 7.52-7.48(\mathrm{~m}, 3 \mathrm{H}, \mathrm{Ar}-\mathrm{H})$, 7.39-7.30 (m, 12H, Ar-H), $5.32(\mathrm{~d}, J=8.3 \mathrm{~Hz}, 1 \mathrm{H}, \mathrm{NH})$, 5.20-5.13 (m, 2H, Bn- $\left.-\mathrm{CH}_{2}\right), 5.11\left(\mathrm{~s}, 2 \mathrm{H}, \mathrm{Bn}-\mathrm{CH}_{2}\right) 4.37(\mathrm{~m}, 1 \mathrm{H}$, $\mathrm{CH}), 4.28\left(\mathrm{t}, J=7.3 \mathrm{~Hz}, 2 \mathrm{H}, \mathrm{CH}_{2}\right), 1.88-1.78\left(\mathrm{~m}, 3 \mathrm{H}, \mathrm{CH}_{2}\right)$ $1.60\left(\mathrm{~m}, 1 \mathrm{H}, \mathrm{CH}_{2}\right)$, and $1.35-1.20\left(\mathrm{~m}, 2 \mathrm{H}, \mathrm{CH}_{2}\right) \cdot{ }^{13} \mathrm{C} \mathrm{NMR}$ $\left(125.7 \mathrm{MHz}, \mathrm{CDCl}_{3}\right): \delta=171.9$ (COO), 155.8 (NCOO), 137.7 (Ar- $\underline{\mathrm{C}}), 136.1$ (Ar- $\underline{\mathrm{C}}), 135.1$ (Ar- $\underline{\mathrm{C}}), 133.0(\underline{\mathrm{CH}}), 129.4$ (Ar- $\underline{\mathrm{CH}})$, 129.1 (2 Ar-CH) 128.8 (12 Ar- $-\mathrm{CH}), 127.1(\mathrm{C}), 67.2\left(\mathrm{CH}_{2}\right), 67.0$ $\left(\underline{\mathrm{CH}}_{2}\right), 53.6(\underline{\mathrm{CH}}), 47.8\left(\underline{\mathrm{CH}}_{2}\right), 32.0\left(\underline{\mathrm{CH}}_{2}\right), 29.5\left(\underline{\mathrm{C}} \mathrm{H}_{2}\right), 22.1$ $\left(\underline{\mathrm{C}} \mathrm{H}_{2}\right) \cdot \mathrm{C}_{29} \mathrm{H}_{30} \mathrm{~N}_{4} \mathrm{O}_{4}(498.23)$. HRMS (ESI-FT-ICR): $m / z=$ 521.21667; calcd. for $\left[\mathrm{C}_{29} \mathrm{H}_{30} \mathrm{~N}_{4} \mathrm{O}_{4} \mathrm{Na}\right]^{+}: m / z=521.21593$.

2.2.2. Benzyl (R)-2-(Benzyloxycarbonylamino)-6-(5-ethoxycarbonyl-1H-1,2,3-triazol-1-yl)hexanoate (10). Colorless oil, yield $65 \mathrm{mg}(52 \%) .[\alpha]_{\mathrm{D}}{ }^{23}=+22.0\left(c=1.5, \mathrm{CHCl}_{3}\right) .{ }^{1} \mathrm{H} \mathrm{NMR}$ $\left(500 \mathrm{MHz}, \mathrm{CDCl}_{3}\right): \delta=8.04(\mathrm{~s}, 1 \mathrm{H}, \mathrm{CH}), 7.39-7.33(\mathrm{~m}, 10 \mathrm{H}$, Ar-H), 5.39 (br d, $1 \mathrm{H}, J=8.3 \mathrm{~Hz}, \mathrm{NH}), 5.22-5.14(\mathrm{~m}, 2 \mathrm{H}$, $\left.\mathrm{Bn}-\mathrm{CH}_{2}\right), 5.11\left(\mathrm{~s}, 2 \mathrm{H}, \mathrm{Bn}-\mathrm{CH}_{2}\right), 4.45-4.30\left(\mathrm{~m}, 3 \mathrm{H}, \mathrm{CH}_{2}, \mathrm{CH}\right)$, $4.32\left(\mathrm{t}, J=7.3 \mathrm{~Hz}, 2 \mathrm{H}, \mathrm{CH}_{2}\right), 1.96-1.86\left(\mathrm{~m}, 3 \mathrm{H}, \mathrm{CH}_{2}\right), 1.73$ $\left(\mathrm{m}, 1 \mathrm{H}, \mathrm{CH}_{2}\right) 1.39\left(\mathrm{t}, J=7.1 \mathrm{~Hz}, 3 \mathrm{H}, \mathrm{CH}_{3}\right), 1.37-1.27(\mathrm{~m}, 2 \mathrm{H}$, $\left.\mathrm{CH}_{2}\right) .{ }^{13} \mathrm{CNMR}\left(125.7 \mathrm{MHz}, \mathrm{CDCl}_{3}\right): \delta=171.8(\mathrm{COO}), 160.7$

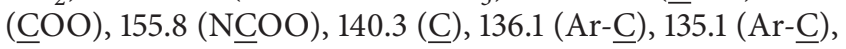
$128.6(10 \mathrm{Ar}-\mathrm{CH}), 127.4(\mathrm{CH}), 67.3\left(\mathrm{CH}_{2}\right), 67.1\left(\mathrm{CH}_{2}\right), 61.3$ $\left(\mathrm{COOCH}_{2}\right), 53.4(\underline{\mathrm{CH}}), 50.2\left(\underline{\mathrm{CH}}_{2}\right), 32.0\left(\underline{\mathrm{CH}}_{2}\right), 29.4\left(\underline{\mathrm{CH}}_{2}\right)$, $21.9\left(\overline{\mathrm{CH}}_{2}\right), 14.3\left(\underline{\mathrm{CH}}_{3}\right) . \mathrm{C}_{26} \mathrm{H}_{30} \mathrm{~N}_{4} \mathrm{O}_{6}(494.22)$. HRMS (ESIFT-ICR): $m / z=517.20527$; calcd. for $\left[\mathrm{C}_{21} \mathrm{H}_{23} \mathrm{NO}_{4} \mathrm{Na}\right]^{+}$: $m / z=517.20567$.

2.3. General Procedure for Wittig Reaction with Nonstabilized Ylids. $n$-Butyl lithium $(0.1 \mathrm{~mL}, 1.6 \mathrm{M}$ in hexane, $0.08 \mathrm{mmol})$ was added to a stirred solution of triphenylalkylphosphonium bromide $(0.08 \mathrm{mmol}, 1$ eq. $)$ in THF $(5 \mathrm{~mL})$, and the resulting mixture was stirred at room temperature for $30 \mathrm{~min}$. The solution of aldehyde $3(25 \mathrm{mg}, 0.07 \mathrm{mmol})$ in THF $(3 \mathrm{~mL})$ was added dropwise to the mixture. The reaction mixture was stirred at room temperature for $1.3 \mathrm{~h}$. After completion of the reaction monitored by TLC (hexane/EE, $7: 3$ ), it was quenched by addition of water and extracted with ether. The combined ether layers were dried and concentrated. The crude product was purified by flash chromatography on silica gel $(n$-hexane/EtOAc $7: 3)$.

2.3.1. Dibenzyl (R)-5-Vinyl-3,4-dihydropyridine-1,2(2H)dicarboxylate (13a). Colorless oil, yield $45.5 \mathrm{mg}(60 \%)$ $[10,11]$.

2.3.2. Dibenzyl (R)-5-(2-Methyl-1-propenyl)-3,4-dihydropyridine-1,2(2H)-dicarboxylate (13b). Colorless oil, yield $105 \mathrm{mg}$ $(65 \%) \cdot[\alpha]_{\mathrm{D}}^{23}=+33.2\left(c=1.5, \mathrm{CHCl}_{3}\right) ;{ }^{1} \mathrm{H} \mathrm{NMR}$ $\left(500 \mathrm{MHz}, \mathrm{CDCl}_{3}\right.$, conformer mixture): $\delta=7.49-7.29$ (m, 10H, Ar-H), 6.94/6.82 (s, 1H, CH), 5.57/5.54 (s, 1H, $\mathrm{CH}), \quad 5.30-5.11\left(\mathrm{~m}, 4 \mathrm{H}, \mathrm{Bn}-\mathrm{CH}_{2}\right), 4.89(\mathrm{~m}, 1 \mathrm{H}, \mathrm{CH})$, $2.45\left(\mathrm{~m}, 1 \mathrm{H}, \mathrm{CH}_{2}\right), 2.12\left(\mathrm{~m}, 1 \mathrm{H}, \mathrm{CH}_{2}\right), 2.03(\mathrm{~m}, 1 \mathrm{H}$, $\left.\mathrm{CH}_{2}\right), 1.95\left(\mathrm{~m}, 1 \mathrm{H}, \mathrm{CH}_{2}\right), 1.80\left(\mathrm{~s}, 3 \mathrm{H}, \mathrm{CH}_{3}\right), 1.77 / 1.88$ (d, $\left.J=5 \mathrm{~Hz}, 3 \mathrm{H}, \mathrm{CH}_{3}\right) \cdot{ }^{13} \mathrm{C} \mathrm{NMR}\left(125.7 \mathrm{MHz}, \mathrm{CDCl}_{3}\right.$, conformer mixture): $\delta=170.8 / 170.6$ (COO), $153.3 / 153.1$ (NCOO), 136.1/135.9 (Ar-C), 135.7/135.5 (Ar-C $), 132.6 / 132.6$ (C) $128.5 / 127.9(10 \mathrm{Ar}-\mathrm{CH}), 124.4 / 124.4(\mathrm{CH}), 122.8 / 122.4$ $(\underline{\mathrm{C}} \mathrm{H}), 116.7 / 116.3(\underline{\mathrm{C}}), 67.9 / 67.7\left(\mathrm{Bn}-\mathrm{CH}_{2}\right), 66.9 / 66.9(\mathrm{Bn}-$ $\left.\underline{\mathrm{CH}}_{2}\right)$, 53.7/53.5 $(\underline{\mathrm{CH}}), 23.8 / 23.6\left(\underline{\mathrm{CH}}_{2}\right), 23.0 / 22.8\left(\underline{\mathrm{CH}}_{2}\right)$, 19.7/19.6 $\left(\mathrm{CH}_{3}\right) . \mathrm{C}_{25} \mathrm{H}_{27} \mathrm{NO}_{4}$ (405.19). HRMS (EI) $\mathrm{m} / z=$ 405.19370; calcd. for $\left[\mathrm{C}_{25} \mathrm{H}_{27} \mathrm{NO}_{4}\right]: m / z=405.19401$.

2.3.3. (R,E)-Dibenzyl 5-(1-Butenyl)-3,4-dihydropyridine1,2(2H)-dicarboxylate and (R,Z)-Dibenzyl 5-(1-Butenyl)-3,4dihydropyridine-1,2(2H)-dicarboxylate (13c). Colorless oil, yield $110 \mathrm{mg}(68 \%),[\alpha]_{\mathrm{D}}{ }^{23}=+31.1\left(c=1.5, \mathrm{CHCl}_{3}\right) ;{ }^{1} \mathrm{H}$ NMR $\left(500 \mathrm{MHz}, \mathrm{CDCl}_{3}, E / Z\right.$ and conformer mixture): $\delta=7.42-7.27(\mathrm{~m}, 10 \mathrm{H}, \operatorname{Ar}-\mathrm{H}), 7.02 / 6.91(\mathrm{~s}, 1 \mathrm{H}, \mathrm{CH})$, $6.07 / 5.99_{\text {trans }}, 5.72 / 5.67_{\text {cis }}\left(2 \mathrm{~d}, J_{\text {trans }}=15.7 \mathrm{~Hz}\right.$ and $2 \mathrm{~d}$, $\left.J_{\text {cis }}=11.6 \mathrm{~Hz}, 1 \mathrm{H}, \mathrm{CH}\right), 5.54-5.11\left(\mathrm{~m}, 5 \mathrm{H}, \mathrm{Bn}-\mathrm{CH}_{2}, \mathrm{CH}\right)$, $4.97(\mathrm{~m}, 1 \mathrm{H}, \mathrm{CH}), 2.45\left(\mathrm{~m}, 1 \mathrm{H}, \mathrm{CH}_{2}\right), 2.31-2.03(\mathrm{~m}, 4 \mathrm{H}$, $\left.\mathrm{CH}_{2}\right), 1.96\left(\mathrm{~m}, 1 \mathrm{H}, \mathrm{CH}_{2}\right), 1.05-0.95\left(\mathrm{~m}, 3 \mathrm{H}, \mathrm{CH}_{3}\right) .{ }^{13} \mathrm{C}$ NMR $\left(125.7 \mathrm{MHz}, \mathrm{CDCl}_{3}\right.$, conformer mixture, signals of both $E$ and $Z$ isomer): $\delta=170.7 / 170.5$ (COO), 153.2/153.0 (NCOO), 135.9/135.8 (Ar-C), 135.6/135.5 (Ar-C), 131.3/131.2 $(\mathrm{C} \overline{\mathrm{H}}) \quad 128.5 / 127.9 \quad(10 \mathrm{Ar}-\mathrm{C}), 127.8 / 127.7 / 127.6 / 127.5(\mathrm{CH})$, $124.7 / 123.6 / 123.2 / 122.7(\underline{\mathrm{CH}})_{\text {cis\&trans }}, 116.6 / 116.2(\underline{\mathrm{C}}), 68.0 / 67.8$ $\left(\mathrm{Bn}-\mathrm{CH}_{2}\right), 67.0 / 66.9\left(\overline{\mathrm{Bn}}-\mathrm{CH}_{2}\right), 53.7 / 53.4(\mathrm{CH}) \quad 23.7 / 23.5$ $\left(\mathrm{CH}_{2}\right), 22.6 / 22.4\left(\underline{\mathrm{CH}}_{2}\right), 22.3 / 22.3\left(\underline{\mathrm{CH}}_{2}\right), 14.9 / 14.8\left(\underline{\mathrm{CH}}_{3}\right)$. $\mathrm{C}_{24} \mathrm{H}_{27} \mathrm{NO}_{4}$ (405.19). HRMS (EI) $m / z=405.19420$; calcd. for $\mathrm{C}_{24} \mathrm{H}_{27} \mathrm{NO}_{4} m / z=405.19401$.

2.3.4. (R,E)-Dibenzyl 5-(1-Propenyl)-3,4-dihydropyridine1,2(2H)-dicarboxylate and (R,Z)-Dibenzyl 5-(1-Propenyl)3,4-dihydropyridine-1,2(2H)-dicarboxylate (13d). Colorless oil, yield $94 \mathrm{mg}(61 \%) \cdot[\alpha]_{\mathrm{D}}^{23}=+30.7\left(c=1.5, \mathrm{CHCl}_{3}\right)$; ${ }^{1} \mathrm{H}$ NMR $\left(500 \mathrm{MHz}, \mathrm{CDCl}_{3}\right.$, conformer mixture, signals of both $E$ and $Z$ isomer): $\delta=7.31-7.16(\mathrm{~m}, 10 \mathrm{H}, \mathrm{Ar}-\mathrm{H})$, 
6.93/6.90/6.81/6.78 (s, 1H, CH), 5.97/5.90 trans, $5.67 / 5.62_{\text {cis }}$ $\left(2 \mathrm{~d}, J_{\text {trans }}=15.5 \mathrm{~Hz}\right.$ and $\left.J_{\text {cis }}=11.7 \mathrm{~Hz}, 1 \mathrm{H}, \mathrm{CH}\right), 5.39$ $(\mathrm{m}, 1 \mathrm{H}, \mathrm{CH}), 5.19-5.00\left(\mathrm{~m}, 4 \mathrm{H}, \mathrm{Bn}-\mathrm{CH}_{2}\right), 4.90(\mathrm{~m}, 1 \mathrm{H}$, $\mathrm{CH}), 2.31\left(\mathrm{~m}, 1 \mathrm{H}, \mathrm{CH}_{2}\right), 2.12\left(\mathrm{~m}, 1 \mathrm{H}, \mathrm{CH}_{2}\right), 2.01(\mathrm{~m}, 1 \mathrm{H}$, $\left.\mathrm{CH}_{2}\right), 1.84\left(\mathrm{~m}, 1 \mathrm{H}, \mathrm{CH}_{2}\right), 1.70-1.61\left(\mathrm{~m}, 3 \mathrm{H}, \mathrm{CH}_{3}\right) .{ }^{13} \mathrm{C} \mathrm{NMR}$ $\left(125.7 \mathrm{MHz}, \mathrm{CDCl}_{3}\right.$, conformer mixture, signals of both $E$ and $Z$ isomer): $\delta=169.6 / 169.4$ (COO), 152.3/152.0 (NCOO), 134.9/134.9 (Ar-ㅡ) , 134.6/134.5 (Ar-C ), 130.0/129.9 ( $\underline{\mathrm{CH}}$ ), 128.6/127.8 (10 Ar-CH), 123.2/122.8/122.1/122.0 ( $\underline{\mathrm{CH}})_{\text {cis\&trans, }}$, $122.0 / 121.5 / 120.1 / 119.8(\underline{\mathrm{CH}})_{\text {cis\&trans }}, 116.0 / 115.2(\underline{\mathrm{C}}), 67.0 / 66.9$ $\left(\mathrm{Bn}-\mathrm{CH}_{2}\right), 65.9 / 65.9\left(\mathrm{Bn}-\mathrm{CH}_{2}\right), 52.8 / 52.4$ ( $\left.\underline{\mathrm{CH}}\right)$ 22.5/22.1 $\left(\underline{\mathrm{CH}}_{2}\right), 21.6 / 21.1\left(\underline{\mathrm{CH}}_{2}\right), 17.5 / 17.2\left(\underline{\mathrm{CH}}_{3}\right) . \mathrm{C}_{24} \mathrm{H}_{25} \mathrm{NO}_{4}$ (391.17). HRMS (EI) $m / z=391.17570$; calcd. for $\left[\mathrm{C}_{24} \mathrm{H}_{25} \mathrm{NO}_{4}\right]$ : $m / z=391.17586$.

2.4. General Procedure for Wittig Reaction with Stable Ylids. The corresponding 2-(triphenylphosphoranylidene)acetate $(0.067 \mathrm{mmol}, 1.8 \mathrm{eq}$. $)$ was added to the solution of protected aldehyde 6 (0.037 mmol, 1 eq., $100 \mathrm{mg})$ in dry toluene $(7 \mathrm{ml})$, and the reaction mixture was refluxed for $18 \mathrm{~h}$. The progress of the reaction was monitored by TLC till the aldehyde was consumed. After completion, the reaction mixture was concentrated and the product was purified chromatographically (pet. Ether/EtOAc $7: 3$ ) to afford the desired product.

2.4.1. Dibenzyl (R,E)-5-(3-Ethoxy-3-oxo-1-propenyl)-3,4-dihydropyridine-1,2(2H)-dicarboxylate (14a). Pale-yellow oil, yield $80 \mathrm{mg}(67 \%) \cdot[\alpha]_{\mathrm{D}}{ }^{23}=+18.2\left(c=1.5, \mathrm{CHCl}_{3}\right) ;{ }^{1} \mathrm{H}$ $\mathrm{NMR}\left(500 \mathrm{MHz}, \mathrm{CDCl}_{3}\right.$, conformer mixture): $\delta=7.46-7.25$ $(\mathrm{m}, 12 \mathrm{H}, \mathrm{Ar}-\mathrm{H}, \mathrm{CH}), 5.68 / 5.67(\mathrm{~d}, J=15.5 \mathrm{~Hz}, 1 \mathrm{CH})$, 5.32-5.10 (m, 4H, Bn- $\left.\mathrm{CH}_{2}\right), 4.99(\mathrm{~m}, 1 \mathrm{H}, \mathrm{CH}), 4.24 / 4.22(\mathrm{q}$, $\left.J=6.8 \mathrm{~Hz}, 2 \mathrm{H}, \mathrm{CH}_{2}\right), 2.52-2.40\left(\mathrm{~m}, 1 \mathrm{H}, \mathrm{CH}_{2}\right), 2.19(\mathrm{~m}$, $\left.1 \mathrm{H}, \mathrm{CH}_{2}\right), 1.99-1.86\left(\mathrm{~m}, 2 \mathrm{H}, \mathrm{CH}_{2}\right), 1.31(\mathrm{t}, J=6.4 \mathrm{~Hz}, 3 \mathrm{H}$, $\left.\mathrm{CH}_{3}\right) .{ }^{13} \mathrm{C} \mathrm{NMR}\left(125.7 \mathrm{MHz}, \mathrm{CDCl}_{3}\right.$, conformer mixture): $\delta=170.0 / 169.9$ (COO), 167.3/167.3 $\left(\underline{\mathrm{COOC}}_{2} \mathrm{H}_{5}\right), 152.8 / 152.8$ (NCOO), 145.1/145.0 ( $\underline{\mathrm{CH}}=\mathrm{CHCOO}), 135.3 / 135.2$ (2 Ar- $\underline{\mathrm{C}}$ ), 132.0/131.6 ( $\underline{\mathrm{CH}}=\mathrm{C}), 128.7$ (10 Ar- $\mathrm{CH}), 115.5 / 115.0(\underline{\mathrm{C}}=\underline{\mathrm{CH}})$, 113.6/113.5 ( $\underline{\mathrm{C}} \mathrm{HCOO}), 68.7 / 68.5\left(\mathrm{Bn}-\mathrm{CH}_{2}\right), 67.3 / 67.2(\mathrm{Bn}-$ $\left.\underline{\mathrm{CH}}_{2}\right), 60.0 / 59.9\left(\underline{\mathrm{CH}}_{2}\right), 54.3 / 53.9(\underline{\mathrm{CH}}), 23.0 / 22.7\left(\underline{\mathrm{CH}}_{2}\right)$, $\overline{18} .0 / 17.8\left(\underline{\mathrm{CH}}_{2}\right), 14 . \overline{3} / 14.2\left(\mathrm{CH}_{3}\right) \cdot \mathrm{C}_{26} \overline{\mathrm{H}}_{27} \mathrm{NO}_{6}$ (449.18). HRMS (EI): $m / z=449.18190$; calcd. for $\mathrm{C}_{26} \mathrm{H}_{27} \mathrm{NO}_{6}: \mathrm{m} / z=$ 449.18384 .

2.4.2. Dibenzyl (R,E)-5-(3-Methoxy-3-oxo-1-propenyl)-3,4-dihydropyridine-1,2(2H)-dicarboxylate (14b). Pale yellow oil, yield $122 \mathrm{mg}(71 \%) .[\alpha]_{\mathrm{D}}{ }^{23}=+16.7\left(c=1.5, \mathrm{CHCl}_{3}\right) ;{ }^{1} \mathrm{H}$ $\mathrm{NMR}\left(500 \mathrm{MHz}, \mathrm{CDCl}_{3}\right.$, conformer mixture): $\delta=7.36-7.15$ $(\mathrm{m}, 12 \mathrm{H}, \mathrm{Ar}-\mathrm{H}, \mathrm{CH}), 5.57 / 5.58(\mathrm{~d}, J=15.4 \mathrm{~Hz}, 1 \mathrm{H}, \mathrm{CH})$, 5.21-5.02 (m, 4H, Bn-CH $)$, 4.96-4.82 (m, $1 \mathrm{H}, \mathrm{CH}), 3.67 / 3.65$ $\left(\mathrm{s}, 3 \mathrm{H}, \mathrm{CH}_{3}\right), 2.42\left(\mathrm{~m}, 1 \mathrm{H}, \mathrm{CH}_{2}\right), 2.12\left(\mathrm{~m}, 1 \mathrm{H}, \mathrm{CH}_{2}\right), 1.89-$ $1.76\left(\mathrm{~m}, 2 \mathrm{H}, \mathrm{CH}_{2}\right) .{ }^{13} \mathrm{C} \mathrm{NMR}\left(125.7 \mathrm{MHz}, \mathrm{CDCl}_{3}\right.$, conformer mixture): $\delta=170.0 / 169.90$ ( $\underline{\mathrm{COO}}), 167.8 / 167.8\left(\mathrm{COOCH}_{3}\right)$, 152.8/152.8 (NCOO), 145.4 ( $\underline{\mathrm{CH}}=\mathrm{CHCOO}), 135.3 / 135.2(2$ Ar-C), $132.2 / 131.8$ ( $\underline{\mathrm{CH}}=\mathrm{C}), 128.7$ (10 Ar-CH), $115.4 / 115.0$ $(\underline{\mathrm{C}}=\mathrm{CH}), 113.1 / 113.0$ ( $\underline{\mathrm{C}} \mathrm{HCOO}), 68.8 / 68.5\left(\mathrm{Bn}-\underline{\mathrm{CH}}_{2}\right), 67.3 / 67.2$ $\left(\overline{\mathrm{Bn}}-\underline{\mathrm{CH}}_{2}\right), 54.3 / 53.9(\underline{\mathrm{CH}}), 51.7 / 51.1\left(\mathrm{CH}_{3}\right), 22.9 / 22.7\left(\underline{\mathrm{CH}}_{2}\right)$, 18.0/17.8 $\left(\mathrm{CH}_{2}\right) \cdot \mathrm{C}_{25} \mathrm{H}_{25} \mathrm{NO}_{6}$ (435.16). HRMS (EI): $\bar{m} / z=$ 435.16780; calcd. for $\mathrm{C}_{25} \mathrm{H}_{25} \mathrm{NO}_{6}: m / z=435.16819$.
2.4.3. Dibenzyl (R,E)-5-(3-Benzyloxy-3-oxo-1-propenyl)-3,4dihydropyridine-1,2(2H)-dicarboxylate (14c). Colorless oil, yield $83 \mathrm{mg}(62 \%) \cdot[\alpha]_{\mathrm{D}}{ }^{23}=+14.9\left(c=1.5, \mathrm{CHCl}_{3}\right) ;{ }^{1} \mathrm{H}$ $\mathrm{NMR}\left(500 \mathrm{MHz}, \mathrm{CDCl}_{3}\right.$, conformer mixture): $\delta=7.49-7.27$ $(\mathrm{m}, 17 \mathrm{H}, \mathrm{Ar}-\mathrm{H}, \mathrm{CH}), 5.75 / 5.76(\mathrm{~d}, J=14.6 \mathrm{~Hz}, 1 \mathrm{H}, \mathrm{CH})$, 5.77-5.12 (m, 6H, Bn- $\left.\mathrm{CH}_{2}\right), 4.98(\mathrm{~m}, 1 \mathrm{H}, \mathrm{CH}), 2.51(\mathrm{~m}, 1 \mathrm{H}$, $\left.\mathrm{CH}_{2}\right), 2.22\left(\mathrm{~m}, 1 \mathrm{H}, \mathrm{CH}_{2}\right), 2.00-1.88\left(\mathrm{~m}, 2 \mathrm{H}, \mathrm{CH}_{2}\right) .{ }^{13} \mathrm{C} \mathrm{NMR}$ $\left(125.7 \mathrm{MHz}, \mathrm{CDCl}_{3}\right.$, conformer mixture): $\delta=170.0 / 169.9$ (COO), 167.2/167.1 ( COOCH$\left._{2} \mathrm{C}_{6} \mathrm{H}_{5}\right), 152.8 / 152.8$ (NCOOO), $145.7 / 145.7$ ( $\mathrm{CH}=\mathrm{CHCOO}), 136.4 / 136.3$ (2 Ar-C), 135.3/135.2 (Ar-C), 132.4/132.0 ( $\mathrm{CH}=\mathrm{C}), 128.7$ (15 Ar- $\overline{\mathrm{CH}}$ ), 115.5/115.0 $(\underline{\mathrm{C}}=\overline{\mathrm{CH}}), 113.2 / 113.1(\underline{\mathrm{CHCOO}}), 68.8 / 68.5\left(\mathrm{Bn}^{-} \underline{\mathrm{CH}}_{2}\right), 67.3 / 67.3$ $\left(\overline{\mathrm{Bn}}-\mathrm{CH}_{2}\right), 66.0 / 65.9\left(\mathrm{Bn}-\mathrm{CH}_{2}\right), 54.3 / 53.9(\overline{\mathrm{CH}}), 23.0 / 22.8$ $\left(\underline{\mathrm{CH}}_{2}\right), 18.1 / 17.8\left(\underline{\mathrm{CH}}_{2}\right) . \mathrm{C}_{31} \mathrm{H}_{29} \mathrm{NO}_{6}$ (511.20). HRMS (EI): $\bar{m} / z=511.20030$; calcd. for $\mathrm{C}_{31} \mathrm{H}_{29} \mathrm{NO}_{6}: m / z=511.19949$.

2.5. General Procedure for the Preparation of Enamino Esters 17. A solution of lithium diisopropylamide $(0.391 \mathrm{mmol}, 2 \mathrm{M}$ in heptanes $)$ in THF $(5 \mathrm{~mL})$ was cooled to $-78^{\circ} \mathrm{C}$. Substituted acetate $(0.778 \mathrm{mmol}, 1$ eq. $)$ in THF $(1 \mathrm{~mL})$ was added dropwise over $30 \mathrm{~min}$, and the solution was allowed to stir for a further $30 \mathrm{~min}$. Protected lactam $16(0.778 \mathrm{mmol}, 1$ eq. $)$ in THF $(2 \mathrm{~mL})$ was added over $30 \mathrm{~min}$ at $-78^{\circ} \mathrm{C}$. Then the solution was allowed to warm up to RT and stirred for $18 \mathrm{~h}$. Satd. aq. $\mathrm{NH}_{4} \mathrm{Cl}$ solution $(10 \mathrm{~mL})$ was added and the mixture was extracted with $\mathrm{CH}_{2} \mathrm{Cl}_{2}(3 \times 100 \mathrm{~mL})$. The combined organic phase was washed with brine $(2 \times 150 \mathrm{~mL})$ and dried over $\mathrm{MgSO}_{4}$, and the solvent was removed in vacuo. TFA $(2.1 \mathrm{~mL}$, $28 \mathrm{mmol}$ ) was added to the crude residue, and the resulting mixture was stirred for $3 \mathrm{~h}$ at $25^{\circ} \mathrm{C}$. Excess TFA was removed in vacuo and the resulting oil was dissolved in $\mathrm{CH}_{2} \mathrm{Cl}_{2}(5 \mathrm{~mL})$. Sat. aq $\mathrm{Na}_{2} \mathrm{CO}_{3}$ was added to neutralize the solution, and the organic components were extracted with $\mathrm{CH}_{2} \mathrm{Cl}_{2}(3 \times 15 \mathrm{~mL})$. The combined organic phase was dried over $\mathrm{MgSO}_{4}$, and the solvent was evaporated in vacuo. The resulting yellow oil was purified by flash column chromatography (n-pentane/EtOAc $4: 1$ ) to give the enamino ester as pale-yellow oil.

2.5.1. Methyl (R,Z)-6-(2-Methoxy-2-oxoethylidene)piperidine2-carboxylate $(\mathbf{1 7 a})$. Yellow oil, yield $55 \mathrm{mg}(67 \%) \cdot[\alpha]_{\mathrm{D}}{ }^{23}=$ $+15.7\left(c=1.5, \mathrm{CHCl}_{3}\right) ;{ }^{1} \mathrm{H} \mathrm{NMR}\left(500 \mathrm{MHz}, \mathrm{CDCl}_{3}\right.$, conformer mixture): $\delta=9.01$ (br s, $1 \mathrm{H}, \mathrm{NH}), 4.48$ (br s, $1 \mathrm{H}, \mathrm{C}=\mathrm{CH}), 4.05(\mathrm{dd}, J=8.0 \mathrm{~Hz}, 5.5 \mathrm{~Hz}, 1 \mathrm{H}, \mathrm{CH}), 3.78(\mathrm{~s}$, $\left.3 \mathrm{H}, \mathrm{COOCH}_{3}\right), 3.63\left(\mathrm{~s}, 3 \mathrm{H}, \mathrm{COOCH}_{3}\right), 2.39-2.36(\mathrm{~m}, 2 \mathrm{H}$, $\left.\mathrm{CH}_{2}\right), 2.19\left(\mathrm{~m}, 1 \mathrm{H}, \mathrm{CH}_{2}\right), 1.90\left(\mathrm{~m}, 1 \mathrm{H}, \mathrm{CH}_{2}\right), 1.79(\mathrm{~m}, 1 \mathrm{H}$, $\left.\mathrm{CH}_{2}\right), 1.68\left(\mathrm{~m}, 1 \mathrm{H}, \mathrm{CH}_{2}\right) .{ }^{13} \mathrm{C} \mathrm{NMR}\left(125 \mathrm{MHz} \mathrm{CDCl}_{3}\right): \delta=$ 172.2 ( $\mathrm{COO}), 170.6(\underline{\mathrm{COO}}), 160.8(\underline{\mathrm{C}}=\mathrm{CH}), 82.7(\mathrm{C}=\underline{\mathrm{CH}}), 53.6$ $(\underline{\mathrm{CH}}), 52.5\left(\mathrm{CH}_{3}\right), 50.0\left(\mathrm{CH}_{3}\right), 28.7\left(\mathrm{CH}_{2}\right), 25.9\left(\mathrm{CH}_{2}\right), 18.7$ $\left(\overline{\mathrm{C}} \mathrm{H}_{2}\right) \cdot \mathrm{C}_{10} \mathrm{H}_{15} \mathrm{NO}_{4}(213.10)$. HRMS (EI) $m / z=213.09978$; calcd. for $\left[\mathrm{C}_{12} \mathrm{H}_{19} \mathrm{NO}_{4}\right]^{+}: m / z=213.09956$.

2.5.2. Methyl (R,Z)-6-(2-Ethoxy-2-oxoethylidene)piperidine2 -carboxylate $(\mathbf{1 7 b})$. Yellow oil, yield $50 \mathrm{mg}(56 \%) \cdot[\alpha]_{\mathrm{D}}{ }^{23}=$ $+14.5\left(c=1.5, \mathrm{CHCl}_{3}\right) ;{ }^{1} \mathrm{H} \mathrm{NMR}\left(500 \mathrm{MHz}, \mathrm{CDCl}_{3}\right.$, conformer mixture): $\delta=9.01$ (br s, $1 \mathrm{H}, \mathrm{NH}), 4.48$ (br s, $1 \mathrm{H}, \mathrm{C}=\mathrm{CH}), 4.10\left(\mathrm{q}, J=7.1 \mathrm{~Hz}, 2 \mathrm{H}, \mathrm{CH}_{2}\right), 4.04(\mathrm{dd}$, $J=8.0 \mathrm{~Hz}, 5.5 \mathrm{~Hz}, 1 \mathrm{H}, \mathrm{CH}), 3.77\left(\mathrm{~s}, 3 \mathrm{H}, \mathrm{CH}_{3}\right), 2.37-2.34$ $\left(\mathrm{m}, 2 \mathrm{H}, \mathrm{CH}_{2}\right), 2.19\left(\mathrm{~m}, 1 \mathrm{H}, \mathrm{CH}_{2}\right), 1.89\left(\mathrm{~m}, 1 \mathrm{H}, \mathrm{CH}_{2}\right), 1.77$ 

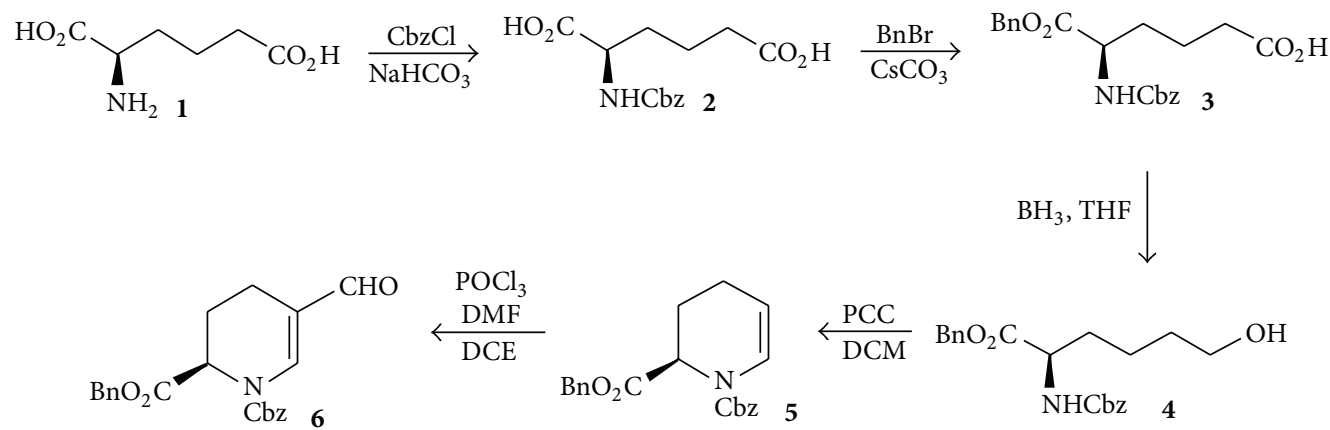

Scheme 1: Synthesis of starting materials (alcohol 4, enamine 5, and aldehyde 6) [10, 11].

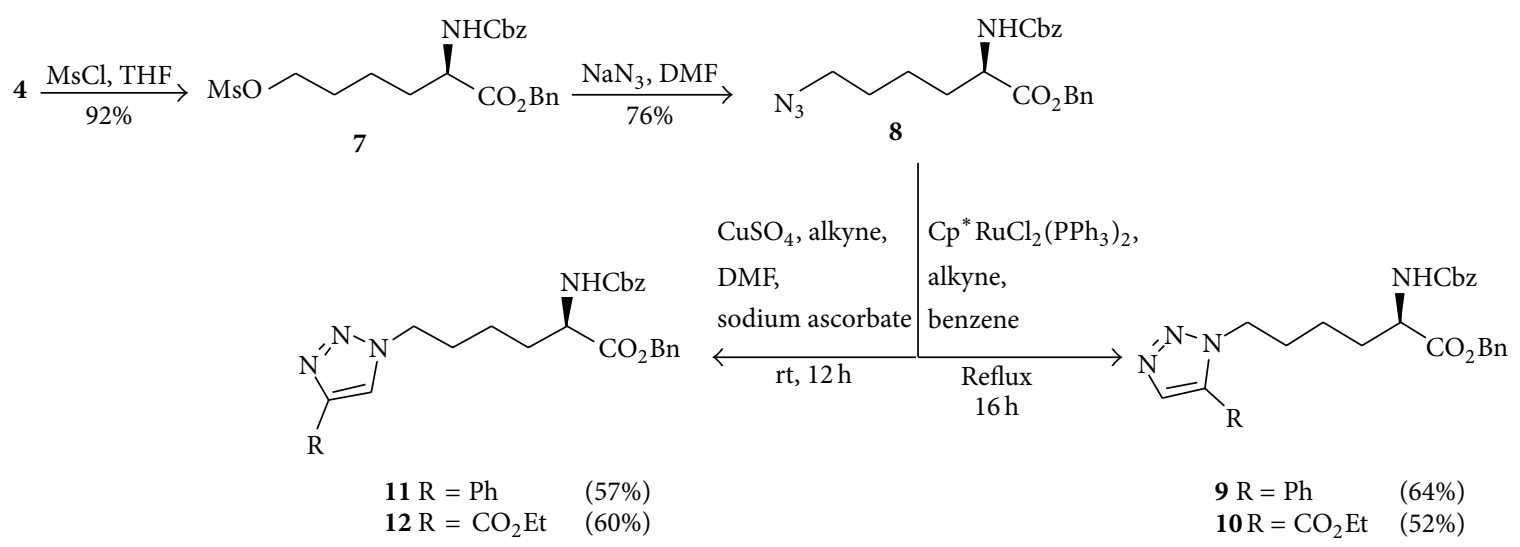

SCHEME 2: Synthesis of azide and triazole derivatives of $(R)$ - $\alpha$-aminoadipic acid.

$\left(\mathrm{m}, 1 \mathrm{H}, \mathrm{CH}_{2}\right), 1.64\left(\mathrm{~m}, 1 \mathrm{H}, \mathrm{CH}_{2}\right), 1.25(\mathrm{t}, \mathrm{J}=7.1 \mathrm{~Hz}, 3 \mathrm{H}$, $\left.\mathrm{CH}_{3}\right) \cdot{ }^{13} \mathrm{C}$ NMR $\left(125 \mathrm{MHz}, \mathrm{CDCl}_{3}\right): \delta=172.2$ ( $\left.\mathrm{COO}\right), 170.3$ $(\underline{\mathrm{COO}}), 160.6(\underline{\mathrm{C}}=\mathrm{CH}), 82.7(\mathrm{C}=\underline{\mathrm{CH}}), 58.4\left(\underline{\mathrm{CH}}_{2}\right), 53.6\left(\underline{\mathrm{CH}}_{3}\right)$, $52.5(\underline{\mathrm{CH}}), 28.8\left(\underline{\mathrm{CH}}_{2}\right), 25.9\left(\underline{\mathrm{CH}}_{2}\right), 18.8\left(\underline{\mathrm{CH}}_{2}\right), 14.6\left(\underline{\mathrm{C}}_{3}\right)$. $\mathrm{C}_{11} \mathrm{H}_{17} \mathrm{NO}_{4}$ (227.12). HRMS (EI) $m / z=227.11548$; calcd. for $\left[\mathrm{C}_{11} \mathrm{H}_{17} \mathrm{NO}_{4}\right]^{+}: m / z=227.11521$.

2.5.3. Methyl (R,Z)-6-(2-tert-Butoxy-2-oxoethylidene)piperidine-2-carboxylate (17c). Yellow oil, yield $65 \mathrm{mg}(66 \%)$. $[\alpha]_{\mathrm{D}}{ }^{23}=+12.9\left(c=1.5, \mathrm{CHCl}_{3}\right) ;{ }^{1} \mathrm{H}$ NMR $(500 \mathrm{MHz}$, $\mathrm{CDCl}_{3}$, conformer mixture): $\delta=8.92$ (br s, $1 \mathrm{H}, \mathrm{NH}$ ), 4.43 (br s, $1 \mathrm{H}, \mathrm{C}=\mathrm{CH}), 4.05(\mathrm{~m}, 1 \mathrm{H}, \mathrm{CH}), 3.79(\mathrm{~s}, 3 \mathrm{H}$, $\left.\mathrm{COOCH}_{3}\right), 2.36-2.32\left(\mathrm{~m}, 2 \mathrm{H}, \mathrm{CH}_{2}\right), 2.12\left(\mathrm{~m}, 1 \mathrm{H}, \mathrm{CH}_{2}\right), 1.87$ $\left(\mathrm{m}, 1 \mathrm{H}, \mathrm{CH}_{2}\right), 1.73\left(\mathrm{~m}, 1 \mathrm{H}, \mathrm{CH}_{2}\right), 1.65\left(\mathrm{~m}, 1 \mathrm{H}, \mathrm{CH}_{2}\right), 1.48$ $\left(\mathrm{s}, 9 \mathrm{H}, \mathrm{C}\left(\mathrm{CH}_{3}\right)_{3}\right) .{ }^{13} \mathrm{C} \mathrm{NMR}\left(125 \mathrm{MHz}, \mathrm{CDCl}_{3}\right): \delta=172.5$

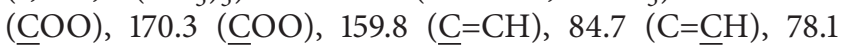

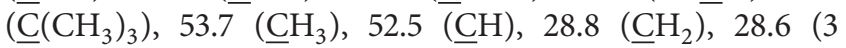
$\left.\underline{\mathrm{C}}\left(\underline{\mathrm{CH}}_{3}\right)_{3}\right), 26.1\left(\underline{\mathrm{C}} \overline{\mathrm{H}}_{2}\right)$, and $18.9\left(\underline{\mathrm{C}} \mathrm{H}_{2}\right) . \mathrm{C}_{13} \mathrm{H}_{21} \mathrm{NO}_{4}$ (255.15). HRMS (EI) $m / \bar{z}=255.14763$; calcd. for $\left[\mathrm{C}_{13} \mathrm{H}_{21} \mathrm{NO}_{4}\right]^{+}$: $m / z=255.14651$.

2.5.4. Ethyl (R,Z)-6-(2-Methoxy-2-oxoethylidene)piperidine2 -carboxylate $(\mathbf{1 7 d})$. Yellow oil, yield $55 \mathrm{mg}(66 \%) \cdot[\alpha]_{\mathrm{D}}{ }^{23}=$ $+13.1\left(c=1.5, \mathrm{CHCl}_{3}\right) ;{ }^{1} \mathrm{H} \mathrm{NMR}\left(500 \mathrm{MHz}, \mathrm{CDCl}_{3}\right.$, conformer mixture): $\delta=9.04$ (br s, $1 \mathrm{H}, \mathrm{NH}), 4.50$ (br s, $1 \mathrm{H}, \mathrm{C}=\mathrm{CH}), 4.13\left(\mathrm{q}, J=7.1 \mathrm{~Hz}, 2 \mathrm{H}, \mathrm{CH}_{2}\right), 4.07(\mathrm{dd}$, $J=8.0 \mathrm{~Hz}, 5.5 \mathrm{~Hz}, 1 \mathrm{H}, \mathrm{CH}), 3.80\left(\mathrm{~s}, 3 \mathrm{H}, \mathrm{CH}_{3}\right), 2.35-2.32$ (m, $\left.2 \mathrm{H}, \mathrm{CH}_{2}\right), 2.16\left(\mathrm{~m}, 1 \mathrm{H}, \mathrm{CH}_{2}\right), 1.88\left(\mathrm{~m}, 1 \mathrm{H}, \mathrm{CH}_{2}\right), 1.75(\mathrm{~m}$, $\left.1 \mathrm{H}, \mathrm{CH}_{2}\right), 1.67\left(\mathrm{~m}, 1 \mathrm{H}, \mathrm{CH}_{2}\right), 1.27 / 1.26(\mathrm{t}, J=7.1 \mathrm{~Hz}, 3 \mathrm{H}$, $\left.\mathrm{CH}_{3}\right) .{ }^{13} \mathrm{C} \mathrm{NMR}\left(125 \mathrm{MHz}, \mathrm{CDCl}_{3}\right): \delta=171.8$ (COO), 170.6 ( $\underline{\mathrm{COO}}), 160.9(\underline{\mathrm{C}}=\mathrm{CH}), 82.2(\mathrm{C}=\underline{\mathrm{CH}}), 61.5\left(\underline{\mathrm{CH}}_{2}\right), 53.7(\underline{\mathrm{CH}})$, $50.0\left(\underline{\mathrm{CH}}_{3}\right), 28.8\left(\underline{\mathrm{CH}}_{2}\right), 25.9\left(\underline{\mathrm{CH}}_{2}\right), 18.7\left(\underline{\mathrm{CH}}_{2}\right), 14.1\left(\underline{\mathrm{CH}}_{3}\right)$. $\mathrm{C}_{11} \mathrm{H}_{17} \mathrm{NO}_{4}$ (227.12). HRMS (EI) $\mathrm{m} / z=227.11521$; calcd. for $\left[\mathrm{C}_{11} \mathrm{H}_{17} \mathrm{NO}_{4}\right]: m / z=227.11548$.

2.5.5. Ethyl (R,Z)-6-(2-Ethoxy-2-oxoethylidene)piperidine-2carboxylate (17e). Yellow oil, yield $47 \mathrm{mg}(53 \%) \cdot[\alpha]_{\mathrm{D}}{ }^{23}=$ $+19.6\left(c=1.5, \mathrm{CHCl}_{3}\right) ;{ }^{1} \mathrm{H} \mathrm{NMR}\left(500 \mathrm{MHz}, \mathrm{CDCl}_{3}\right.$, conformer mixture): $\delta=9.01$ (br s, $1 \mathrm{H}, \mathrm{NH}$ ), 4.48 (br s, $1 \mathrm{H}$, $\mathrm{C}=\mathrm{CH}), 4.24\left(\mathrm{q}, J=7.1 \mathrm{~Hz}, 2 \mathrm{H}, \mathrm{CH}_{2}\right), 4.12(\mathrm{q}, J=7.1 \mathrm{~Hz}$, $2 \mathrm{H}, \mathrm{CH}_{2}$ ), 4.03 (dd, $\left.J=8.0 \mathrm{~Hz}, 5.4 \mathrm{~Hz}, 1 \mathrm{H}, \mathrm{CH}\right), 2.36-2.32$ $\left(\mathrm{m}, 2 \mathrm{H}, \mathrm{CH}_{2}\right), 2.15\left(\mathrm{~m}, 1 \mathrm{H}, \mathrm{CH}_{2}\right), 1.84\left(\mathrm{~m}, 1 \mathrm{H}, \mathrm{CH}_{2}\right), 1.75$ $\left(\mathrm{m}, 1 \mathrm{H}, \mathrm{CH}_{2}\right), 1.67\left(\mathrm{~m}, 1 \mathrm{H}, \mathrm{CH}_{2}\right), 1.30(\mathrm{t}, J=7.1 \mathrm{~Hz}, 3 \mathrm{H}$, $\left.\mathrm{CH}_{3}\right), 1.26\left(\mathrm{t}, \mathrm{J}=7.1 \mathrm{~Hz}, 3 \mathrm{H}, \mathrm{CH}_{3}\right) .{ }^{13} \mathrm{C} \mathrm{NMR}(125 \mathrm{MHz}$, $\left.\mathrm{CDCl}_{3}\right): \delta=170.8(\underline{\mathrm{COO}}), 170.3(\underline{\mathrm{COO}}), 160.7(\underline{\mathrm{C}}=\mathrm{CH})$, $82.7(\mathrm{C}=\underline{\mathrm{CH}}), 61.5\left(\underline{\mathrm{CH}}_{2}\right), 58.4(\underline{\mathrm{CH}}), 53.7\left(\underline{\mathrm{CH}}_{2}\right), 28.8\left(\mathrm{CH}_{2}\right)$, $26.0\left(\mathrm{CH}_{2}\right), 18.8\left(\overline{\mathrm{CH}}_{2}\right), 14.6\left(\underline{\mathrm{CH}}_{3}\right), 14.1\left(\underline{\mathrm{CH}}_{3}\right)$. HRMS (EI) $m / z=241.13120$; calcd. for $\left[\mathrm{C}_{12} \mathrm{H}_{19} \mathrm{NO}_{4}\right]: m / z=241.13141$.

\section{Results and Discussion}

We previously disclosed the syntheses of alcohol 4, enamine 5 , and aldehyde 6 from $(R)$ - $\alpha$-aminoadipic acid (Scheme 1 ) $[10,11]$. 
<smiles>[R]OC(=O)C=CC1=CN([14CH3])[C@H](C(=O)O)CC1</smiles>

14a $\mathrm{R}=\mathrm{C}_{2} \mathrm{H}_{5} \quad(67 \%)$ 14b $\mathrm{R}=\mathrm{CH}_{3} \quad(71 \%)$ 14c $\mathrm{R}=\mathrm{C}_{6} \mathrm{H}_{5} \mathrm{CH}_{2} \quad$ (62\%)

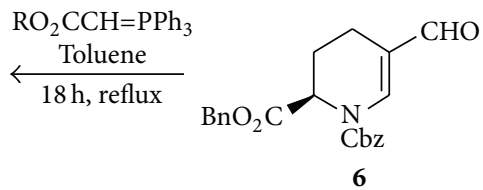<smiles>[R]C([R])=CC1=CN([R10])[C@H](C(=O)OBr)CC1</smiles>

13a $\mathrm{R}=\mathrm{H}, \mathrm{R}^{\prime}=\mathrm{H}$ 13b R $=\mathrm{CH}_{3}, \mathrm{R}^{\prime}=\mathrm{CH}_{3}$ $13 \mathrm{c} \mathrm{R}=\mathrm{C}_{3} \mathrm{H}_{7}, \mathrm{R}^{\prime}=\mathrm{H}$ 13d R $=\mathrm{C}_{2} \mathrm{H}_{5}, \mathrm{R}^{\prime}=\mathrm{H}$
$(60 \%)$

$(65 \%)$

$(68 \%, \mathrm{E} / \mathrm{Z})$

$(61 \%, E / Z)$

SCHEME 3: Synthesis of C-5-alkenyl derivatives of $(R)$-pipecolic acid.

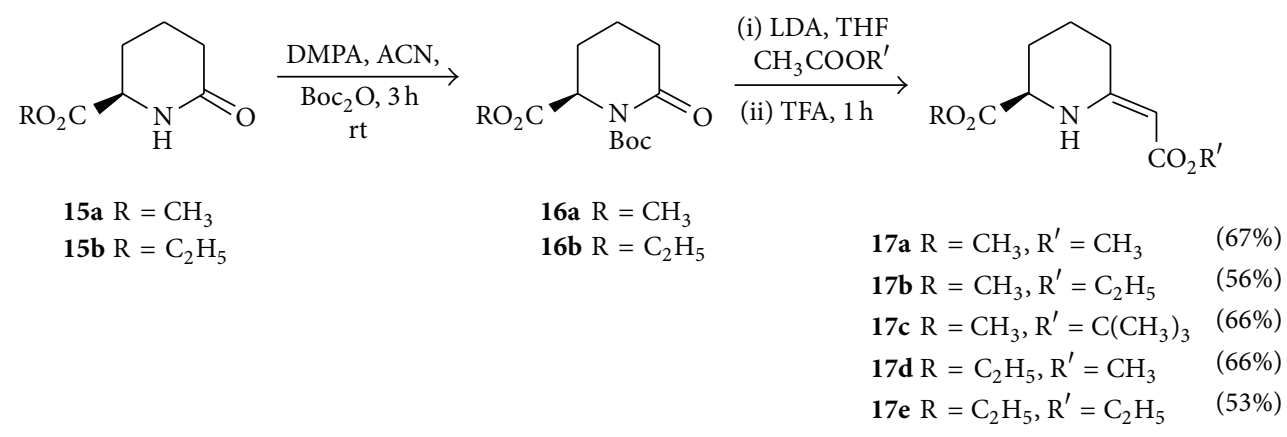

SCHEME 4: Synthesis of C-6 enamino esters of (R)-pipecolic acid.

Amino acids with azido functions in the side chain are appreciated in organic synthesis for its ease of introduction into complex structures, convenient conversion to a primary amine [22], and participation in dipolar cycloaddition reactions [23], especially with respect to bioorthogonal reactions [24]. For the synthesis of the azide $\mathbf{8}$, alcohol $\mathbf{4}$ was first converted into the methane sulfonate 7 using methanesulfonyl chloride and triethylamine. It was then converted into azide $\mathbf{8}$ by treatment with sodium azide in DMF at room temperature.

Keeping in view the importance of triazole system [25] and with this azide intermediate in our hands, we performed click reactions using $\mathrm{CuSO}_{4} /$ ascorbate and $\mathrm{Cp}{ }^{*} \mathrm{Ru}\left(\mathrm{PPh}_{3}\right)_{2} \mathrm{Cl}_{2}$ as catalysts for the synthesis of 1,4- and 1,5-disubstituted triazole amino acid derivatives. Treatment of azide $\mathbf{8}$ with alkynes proceeded smoothly in the presence of a catalytic amount of $\mathrm{CuSO}_{4}$ and sodium ascorbate in a $10 \%$ solution of DMSO in water to produce 1,4-disubstituted-1,2,3-triazole derivatives $\mathbf{1 1}$ and $\mathbf{1 2}$ in good yields, respectively. The synthesis of 1,5-disubstituted triazole regioisomers was carried out successfully under ruthenium catalysis in good yields by refluxing azide $\mathbf{8}$ and catalyst and alkyne in benzene (Scheme 2).

Aldehyde moieties present in amino acids constitute a class of chiral synthons, valuable for the synthesis of optically active compounds and, in particular, for the synthesis of unusual amino acids. Thus, for the synthesis of C-5-alkenyl derivatives of $(R)$-pipecolic acid, formylation of enamine $\mathbf{5}$ by Vilsmeier-Haack reaction under reflux conditions was performed to provide protected aldehyde 6 (Scheme 1). One example of a Wittig reaction of aldehyde $\mathbf{6}$ giving a vinyl derivative of pipecolic acid has already been described by us [12]. In order to explore the scope of the reaction, aldehyde
6 was treated with a range of Wittig reagents to afford C5 -alkenyl derivatives of $(R)$-pipecolic acid. The reaction was carried out with nonstabilized ylids generated in situ from alkyl triphenylphosphonium bromides with $n$-BuLi at $-78^{\circ} \mathrm{C}$ to afford the alkenyl substituted products in good yield. Compounds $13 \mathrm{c}$ and $\mathbf{1 3 d}$ were obtained as a mixture of $\mathrm{E} / \mathrm{Z}$ isomers (Scheme 3; 13a-d).

Wittig reaction of aldehyde $\mathbf{6}$ was also investigated with an array of stabilized ylids that are preformed and added to suitable carbonyl compounds. This reaction was performed in toluene as a solvent under refluxing conditions for 18 hours and provided exclusively $E$-configured $\alpha, \beta$-unsaturated ester derivatives of pipecolic acid 14 (Scheme 3, 14a-c).

Strongly acidic or basic reaction conditions often lead to epimerization or racemization in susceptible chiral compounds. Therefore, the enantiomeric purity of the reaction products 6 and 13a was determined by chiral HPLC (Chiralpak-AD).

For comparison the $S$-configured enantiomers ent-6 and ent-13a were synthesized starting from $(S)$ - $\alpha$-aminoadipic acid and analyzed as well. No epimerization could be detected for the two compounds investigated (Figure 1).

$(R)-6-O x o p i p e c o l i c$ acid derivatives 15 can be easily prepared from $(R)$ - $\alpha$-aminoadipic acid in consecutive steps of esterification and cyclization during Kugelrohr distillation according to previously reported procedures $[12,26]$. The amide group of $\mathbf{1 5}$ was Boc-protected with Boc anhydride and DMAP in acetonitrile at room temperature. For synthesis of C6-exocyclic enamino esters of pipecolic acid, compound 16 was reacted with a variety of substituted alkyl acetates in presence of LDA followed by treatment with TFA for $2 \mathrm{~h}$ at room temperature to give the desired compounds 


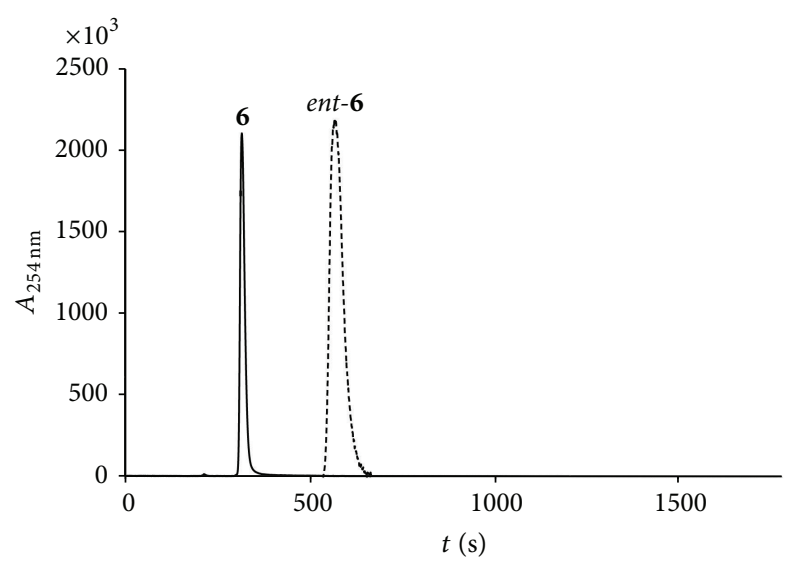

(a)

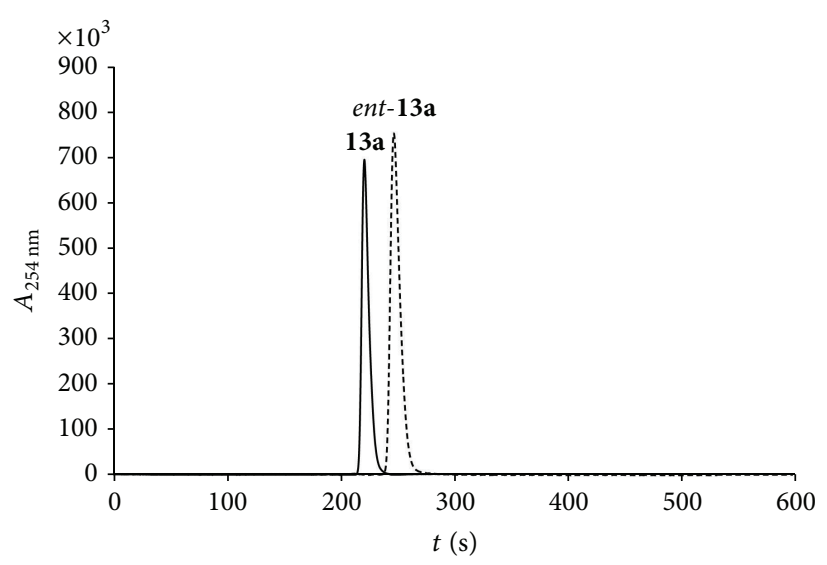

(b)

Figure 1: (a) Determination of enantiomeric purities by chiral HPLC analysis of $\mathbf{6} .6 t_{R}=5.26 \mathrm{~min}$, ent-6 $t_{R}=9.48 \mathrm{~min}$. (b) Determination of enantiomeric purities by chiral HPLC analysis of 13a. 13a $t_{R}=3.67 \mathrm{~min}$, ent $-13 \mathrm{a} t_{R}=4.20 \mathrm{~min}$.

(Scheme 4). The $Z$ configuration of the synthesized compounds was established on the basis of chemical shift values of the alkenyl and the NH protons. The chemical shift values for these protons are significantly deshielded which indicates the $Z$ configuration, and this is in good agreement with the data reported for 5-membered cyclic analogs of these compounds [27].

In summary, efficient and simple syntheses of C-5 alkenyl and C- 6 enamino ester derivatives of pipecolic acid are presented from enantiopure $(R)$ - $\alpha$-aminoadipic acid. Furthermore, the 6-azido and regioisomeric 6-triazolyl derivatives of amino $(R)-\alpha$-aminohexanoic acid were prepared starting from $(R)-\alpha$-aminoadipic acid by $\mathrm{CuAAC}$ or RuAAC.

\section{Acknowledgments}

The authors gratefully acknowledge the German Academic Exchange Service DAAD for the award of a Ph.D. fellowship to Amina Sadiq. Sandoz GmbH, Kundl, Austria, and Trend Materials $\mathrm{GmbH}$, Linz, Austria, supported the project by providing the starting material $(R)$ - $\alpha$-aminoadipic acid.

\section{References}

[1] J. F. Sardina and H. Rapoport, "Enantiospecific synthesis of heterocycles from $\alpha$-amino acids," Chemical Reviews, vol. 96, no. 6, pp. 1825-1872, 1996.

[2] F. P. J. T. Rutjes, L. B. Wolf, and H. E. Schoemaker, "Applications of aliphatic unsaturated non-proteinogenic $\alpha$-H- $\alpha$-amino acids," Journal of the Chemical Society, Perkin Transactions 1, no. 24, pp. 4197-4212, 2000.

[3] J. Kaiser, S. S. Kinderman, B. C. J. van Esseveldt et al., "Synthetic applications of aliphatic unsaturated $\alpha$-H- $\alpha$-amino acid," Organic and Biomolecular Chemistry, vol. 3, no. 19, pp. 3445-3467, 2005.

[4] S. R. Gracia, K. Gaus, and N. Sewald, "Synthesis of chemically modified bioactive peptides: recent advances, challenges and developments for medicinal chemistry," Future Medicinal Chemistry, vol. 1, no. 7, pp. 1289-1310, 2009.
[5] X. Zhu, G. Tang, and G. Galili, "The catabolic function of the $\alpha$-aminoadipic acid pathway in plants is associated with unidirectional activity of lysine-oxoglutarate reductase, but not saccharopine dehydrogenase," Biochemical Journal, vol. 351, no. 1, pp. 215-220, 2000.

[6] H. McLennan, T. P. Hicks, and J. R. Liu, "On the configuration of the receptors for excitatory amino acids," Neuropharmacology, vol. 21, no. 6, pp. 549-554, 1982.

[7] J. W. Olney, J. Labruyere, J. F. Collins, and K. Curry, "DAminaphosphonovalerate is 100 -fold more powerful than D$\alpha$-aminoadipate in blocking $\mathrm{N}$-methylaspartate neurotoxicity," Brain Research, vol. 221, no. 1, pp. 207-208, 1981.

[8] S. C. Bergmeier, A. A. Cobls, and H. Rapoport, "Chirospecific synthesis of (1S,3R)-1-amino-3-(hydroxymethyl)cyclopentane, precursor for carbocyclic nucleoside synthesis. Dieckmann cyclization with an $\alpha$-amino acid," Journal of Organic Chemistry, vol. 58, no. 9, pp. 2369-2376, 1993.

[9] V. C. Sonawane, "Enzymatic modifications of cephalosporins by cephalosporin acylase and other enzymes," Critical Reviews in Biotechnology, no. 2, pp. 95-120, 2006.

[10] A. Sadiq and N. Sewald, "(R)- $\alpha$-aminoadipic acid: an interesting chiral pool building block," Arkivoc, vol. 2012, no. 5, pp. 28-36, 2012.

[11] A. Sadiq and N. Sewald, "6-Alkynyl- and 6-aryl-substituted (R)pipecolic acid derivatives," Organic Letters, vol. 15, no. 11, pp. 2720-2722, 2013.

[12] T. D. Copeland, E. M. Wondrak, J. Tozser, M. M. Roberts, and S. Oroszlan, "Substitution of proline with pipecolic acid at the scissile bond converts a peptide substrate of HIV proteinase into a selective inhibitor," Biochemical and Biophysical Research Communications, vol. 169, no. 1, pp. 310-314, 1990.

[13] T. B. A. Ekenstam and C. Bovin, "L-N-n-Propylpipecolic acid2,6-xylidide," US Patent 4 695 576/1987 (Chem. Abstr., 1988, 108, 57228n).

[14] R. Kikumoto, Y. Tamao, T. Tezuka et al., "Selective inhibition of thrombin by $(2 \mathrm{R}, 4 \mathrm{R})$-4-methyl-1-[N2-[1,2,3,4-tetrahydro8-quinolinyl)sulfonyl]-L-arginyl]-2-piperidinecarboxylic acid," Biochemistry, vol. 23, no. 1, pp. 85-90, 1984.

[15] A. G. Flynn, L. E. Giruox, and C. R. Dage, "An acyl-iminium ion cyclization route to a novel conformationally restricted dipeptide mimic: applications to angiotensin-converting enzyme 
inhibition," Journal of the American Chemical Society, vol. 109, no. 25, pp. 7914-7917, 1987.

[16] G. J. Gatto Jr., M. T. Boyne II, N. L. Kelleher, and C. T. Walsh, "Biosynthesis of pipecolic acid by RapL, a lysine cyclodeaminase encoded in the rapamycin gene cluster," Journal of the American Chemical Society, vol. 128, no. 11, pp. 3838-3847, 2006.

[17] M. Nakatsuka, J. A. Ragan, T. Sammakia, D. B. Smith, D. E. Uehling, and S. L. Schreiber, "Total synthesis of FK506 and an FKBP probe reagent, (C8,C9-13C2)-FK506," Journal of the American Chemical Society, vol. 112, no. 14, pp. 5583-5601, 1990.

[18] D. L. Boger, J. Chen, and K. W. Saionz, "(-)-Sandramycin: total synthesis and characterization of DNA binding properties," Journal of the American Chemical Society, vol. 118, no. 7, pp. 1629-1644, 1996.

[19] U. A. Germann, D. Shlyakhter, V. S. Mason et al., "Cellular and biochemical characterization of VX-710 as a chemosensitizer: reversal of P-glycoprotein-mediated multidrug resistance in vitro," Anti-Cancer Drugs, vol. 8, no. 2, pp. 125-140, 1997.

[20] S. Weigelt, T. Huber, F. Hofmann et al., "Synthesis and conformational analysis of efrapeptins," Chemistry, vol. 18, no. 2, pp. 478-487, 2012.

[21] T. A. Miller, D. J. Witter, and S. Belvedere, "Histone deacetylase inhibitors," Journal of Medicinal Chemistry, vol. 46, no. 24, pp. 5097-5116, 2003.

[22] E. Scriven and K. Turnbull, "Azides: their preparation and synthetic uses," Chemical Reviews, vol. 88, no. 2, pp. 296-368, 1988.

[23] A. R. Katritzky, Y. Zhang, and S. K. Singh, "1,2,3-Triazole formation under mild conditions via 1,3-dipolar cycloaddition of acetylenes with azides," Heterocycles, vol. 60, no. 5, pp. 12251239, 2003.

[24] Y. L. Angell and K. Burgess, "Peptidomimetics via coppercatalyzed azide-alkyne cycloadditions," Chemical Society Reviews, vol. 36, no. 10, pp. 1674-1689, 2007.

[25] M. Gajewski, B. Seaver, and S. C. Esslinger, "Design, synthesis, and biological activity of novel triazole amino acids used to probe binding interactions between ligand and neutral amino acid transport protein SN1," Bioorganic and Medicinal Chemistry Letters, vol. 17, no. 15, pp. 4163-4166, 2007.

[26] S. B. Huang, J. S. Nelson, and D. D. Weller, "Preparation of optically pure $\omega$-hydroxymethyl lactams," Synthetic Communications, vol. 19, no. 20, pp. 3485-3496, 1989.

[27] M. C. Elliott and S. V. Wordingham, "A convenient protocol for the alkylidenation of lactams," Synthesis, no. 7, pp. 1162-1170, 2006. 

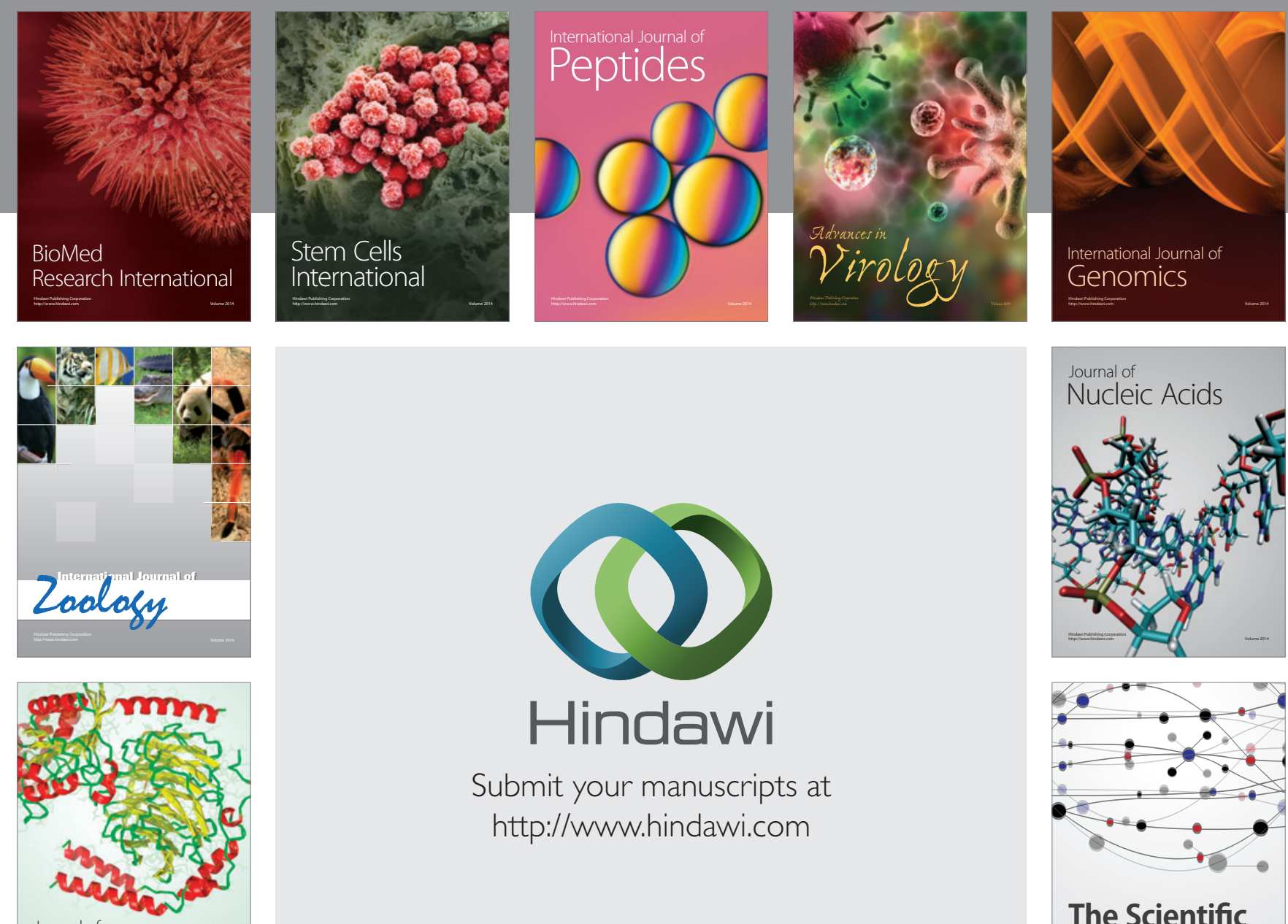

Submit your manuscripts at

http://www.hindawi.com

Journal of
Signal Transduction
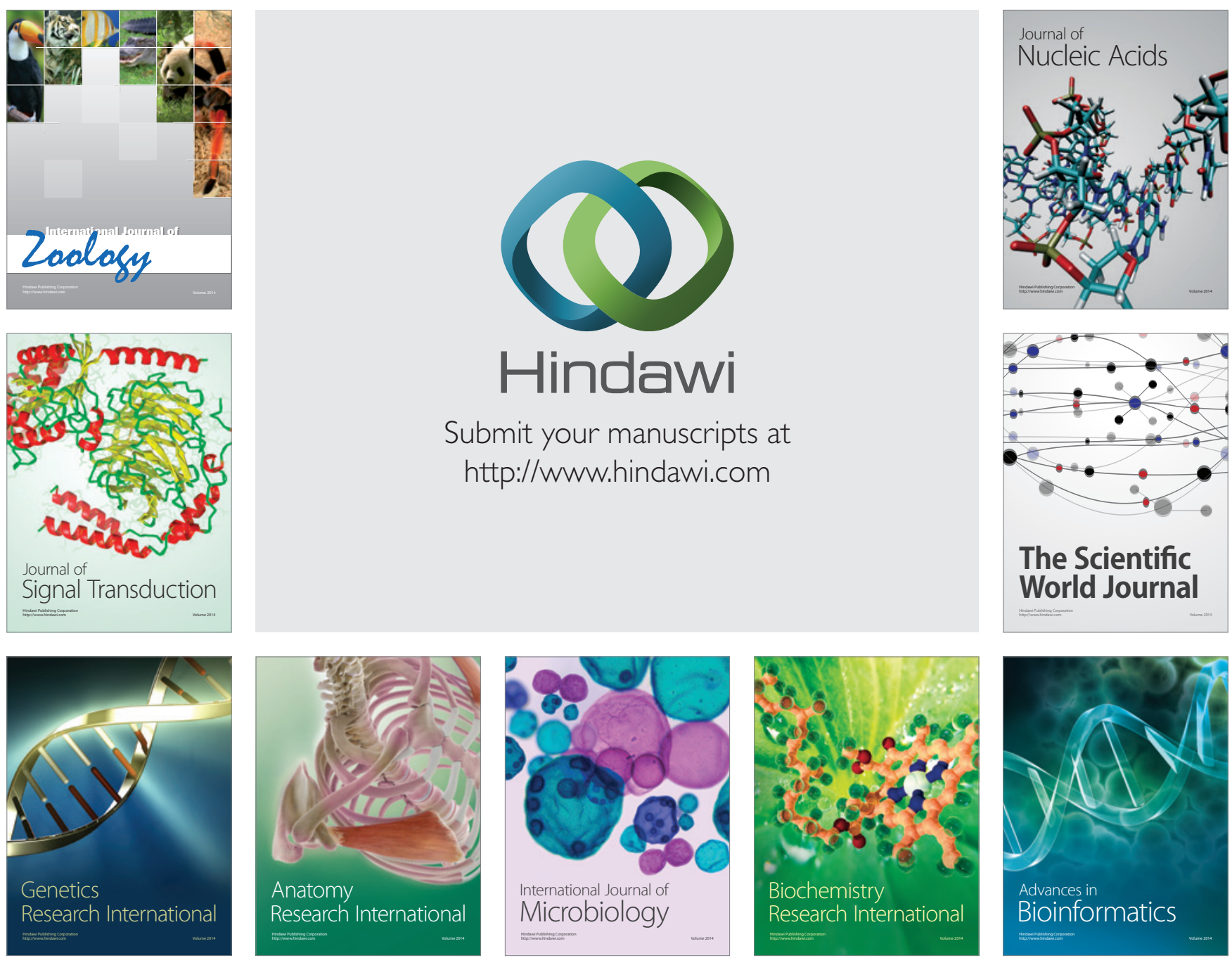

The Scientific World Journal
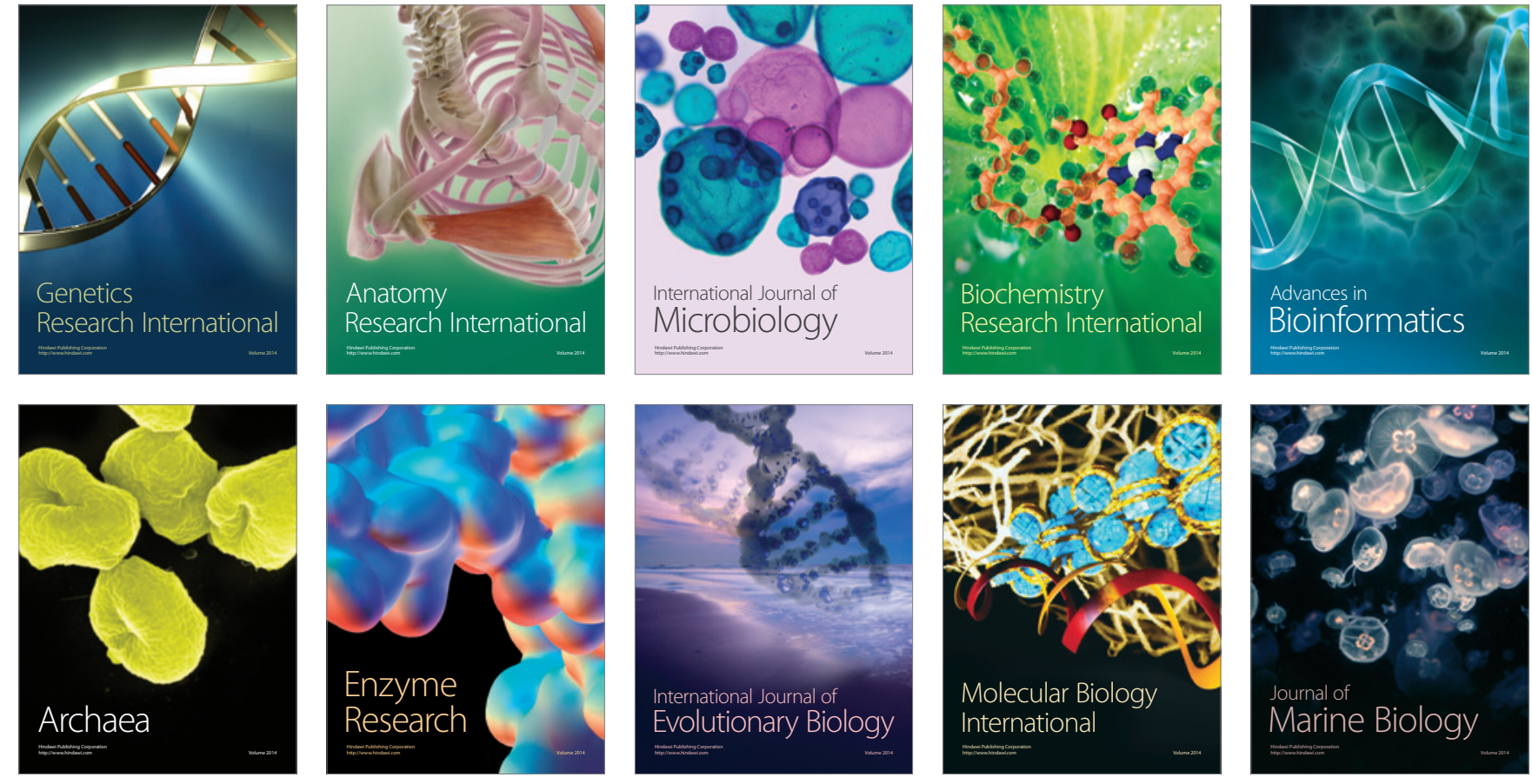